\title{
Experimental and numerical investigation of blade resonance in a centrifugal compressor for varying gas properties
}

\section{Original article}

Article history:

Accepted: 20 June 2018

Published: 20 September 2018

\section{Check for updates}

\section{${ }^{*}$ Correspondence:}

CD: degendorfer@lec.mavt.ethz.ch

\section{Peer review:}

Single blind

\section{Copyright:}

(c) 2018 Degendorfer et al. () This is an open access article distributed under the Creative Commons Attribution Non Commercial No Derivatives License (CC BY-NC-ND 4.0). Unrestricted use, distribution, and reproduction of the original work are permitted for noncommercial purposes only, provided it is properly cited and its authors credited. No derivative of this work may be distributed.

\section{Keywords:}

fluid-structure-interaction; generalized force; blade damping; centrifugal compressor; carbon dioxide; blade resonance

\section{Citation:}

Degendorfer C., Abhari R. S., Vogel K., and Hunziker R. (2018). Experimental and numerical investigation of blade resonance in a centrifugal compressor for varying gas properties. Journal of the Global Power and Propulsion Society. 2: 415-428. https://doi.org/10.22261/JGPPS.Q15CRP

\author{
Carsten Degendorfer ${ }^{1,{ }^{*}}$, Reza S. Abhari $^{1}$, Klemens Vogel $^{2}$, René Hunziker $^{2}$ \\ ${ }^{1}$ Laboratory for Energy Conversion, ETH Zurich, Department of Mechanical and Process \\ Engineering, Sonneggstrasse 3, CH-8092 Zurich, Switzerland \\ ${ }^{2}$ ABB Turbo Systems Ltd., Bruggerstrasse 71a, CH-5401 Baden, Switzerland
}

\begin{abstract}
The blades of centrifugal compressors are exposed to unsteady forces during operation which can result in resonance response conditions and failures due to high cycle fatigue. A typical source of excitation is the unsteady fluid structure interaction between the impeller blades and the downstream vaned diffuser. Centrifugal compressors are operated with various working fluids with a wide range of applications in the power and process industry. Understanding the excitation mechanisms for different working fluids will help to design aerodynamically efficient compressors, while ensuring mechanical integrity and reducing the number of experimental design validations. A variation in working fluid properties allows investigation of the contribution of blade forcing and damping while the modal response remains unchanged. Experiments have been conducted at ETH Zurich's radial compressor facility with a state of the art industrial compressor design. Dynamic strain gauge measurements on the impeller blades were used to determine the amplitude response, damping properties and forcing at a defined resonance condition. Two working fluids have been investigated to vary compressor flow settings while the modal response remains unchanged. Unsteady flow simulations and harmonic FSI simulations were used to complement the experiments and to investigate the local blade forcing distribution, which then were linked to flow effects. Experiments showed a change in resonance amplitude up to a factor of 4 due to a change in the applied working fluid. Estimation of the damping ratio with a single degree of freedom model found the exciting force to be the main contributor to the differences in resonant response. The unsteady flow simulations were able to identify the locations on the blade surface which are responsible for the change in forcing. It was found that the forcing depends on wave propagation effects in the flow channel and on how the pressure field matches the mode shape.
\end{abstract}

\section{Introduction}

Radial compressors are found in a diverse range of applications and are operated with many different working fluids. The demand for aerodynamic performance and efficiency results in high material load and induced vibrations. Resonance on the rotating blades leads to high cycle fatigue and is one of the main contributors to failures (Srinivasan, 1997). It is therefore required to assess vibrations and high cycle fatigue mechanisms during the design phase of the components since they contribute to the development and maintenance costs (El-Aini et al., 1997; Kielb, 1998). 
Among other sources of excitation, fluid structure interaction between the impeller blades and the downstream vaned diffuser is of significance as showed by (Haupt and Rautenberg, 1984). Past research on impeller-diffuser interaction (IDI) is often focused on the impact of geometrical design variations. For example experimental flow investigations have been undertaken by (Ziegler et al., 2003a,b) where a small radial gap between impeller exit and diffuser inlet lead to more homogenous flow structures, and according to (Gallier et al., 2010) it also leads to reduced wake regions at impeller exit. Time resolved flow simulations of radial compressors have been conducted by (Boncinelli et al., 2007) showing that smaller radial gap sizes are beneficial to the compressor performance. More recent studies on the influence of the radial gap size on resonant blade response have been conducted by (Zemp and Abhari, 2012). Experimental and numerical investigations showed increased unsteady forces and the highest vibratory response amplitude for small radial gaps. Damping and response characteristics were found to be highly dependent on blade mode and operating points. It has been shown that numerical flow simulations alone are not sufficient to predict response behaviour.

A numerical study investigating the impact of disk and blade geometry on mode coupling was conducted by (Walton and Tan, 2016). The generalized force, obtained by unsteady CFD simulations, and mode shape, was used as input for a two mass-spring system to estimate the amplitude response. The results indicate that the generalized force is able to describe blade excitation mechanisms.

In the area of vibration in process compressors, research has focused on acoustic modes generated in the compressor and causing fatigue of the connected piping system ( $\mathrm{Li}$ et al., 2015) but not on resonant blade vibration. In the publication of (König et al., 2009) the cause of two impeller failures for high pressure natural gas compressors have been investigated and found to be caused by fluid structure interaction.

To the best of the author's knowledge, no study has been undertaken that provides a direct comparison in terms of vibratory response for two working fluids of a compressor stage while the modal response remains unchanged. Due to the high sensitivity of blade excitation to flow and forcing, an investigation was found to be beneficial for future compressor design optimizations which would aim on minimizing the blade forcing. Within the scope of this study, $\mathrm{CO}_{2}$ was chosen for comparison with air. $\mathrm{CO}_{2}$ possesses distinct gas properties while allowing safe operation of the facility. Additionally, compression of $\mathrm{CO}_{2}$ will have increased relevance for carbon capture and storage (CCS) application in near zero emission power plants (Metz et al., 2005). Publications in this field were found to focus on efficiency improvements of the capturing process (Botero et al., 2009; Al-Abdulkarem et al., 2012). Further applications for $\mathrm{CO}_{2}$ are found in super critical power cycles with on-going research in efficiency and performance, e.g. (Heo et al., 2017) and fluid structure interaction of a simplified impeller like disk setup (Pereboom et al., 2016).

\section{Methodology}

The goal of this paper is to discuss the impact of forcing and damping on the resonance response amplitudes of a radial compressor for two different working fluids.

Dynamic strain gauges on the impeller main blade are used to measure the resonance crossing of mode 4, excited by the downstream vaned diffuser. Capturing the resonance crossing allows determining the maximum strain as well as estimation of the system's critical damping ratio. This information is sufficient to determine the contribution of unsteady blade forcing to the operating point dependent resonant response amplitudes.

Unsteady CFD and FSI simulation approaches are used to complement the experiments. The generalized force distribution on the impeller blade is calculated based on time dependent blade pressure data obtained by CFD simulations. The results allow investigation of the blade regions that are most affected by changes in blade forcing.

Additional FSI simulations, using CFD and damping information from strain gauge (SG) measurements as input, are used to estimate the validity of the CFD simulations.

\section{Facility overview}

The experiments have been conducted at the 'RIGI' centrifugal compressor facility at the Laboratory for Energy Conversion at the Swiss Federal Institute of Technology in Zurich, Switzerland. A schematic of the test rig is shown in Figure 1 and is operated in a closed loop configuration, which allows controlling the stage inlet pressure, temperature and working fluid composition independently from the ambient conditions. The compressor is driven by a $485 \mathrm{~kW}$ electric motor allowing a maximum shaft speed of 22,000 rpm. Downstream of the compressor the working fluid is cooled by a heat exchanger. A variable throttle is used to discharge the flow and to set the desired mass flow rate, which is measured by a standard orifice. Pressure and temperature sensors up and downstream the compressor stage are used to estimate the compressor performance. The upstream pressure is 


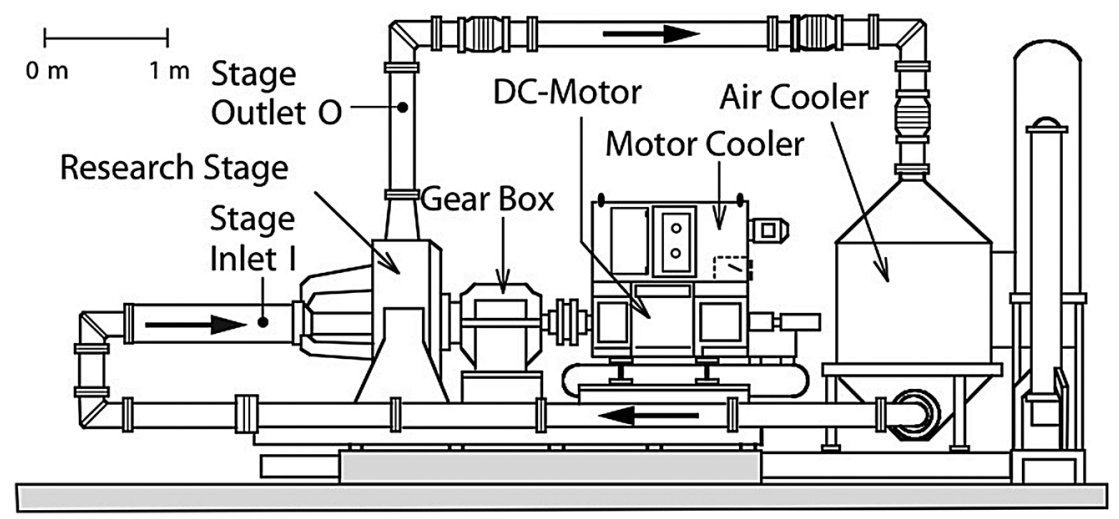

Figure 1. Centrifugal compressor facility overview.

actively controlled by a gas extraction and injection system. This in-house developed system is also responsible for controlling the $\mathrm{CO}_{2}$ concentration of the working fluid and measuring it with a time of flight sensor. The sensor is temperature compensated and calibrated to ambient air before each measurement run. Measurement accuracy of the $\mathrm{CO}_{2}$ concentration is $1.4 \mathrm{wt} \%$, estimated by the method presented in (GUM, 1995).

The maximum inlet pressure, and density of the stage is limited by the available power of the electric motor and is below atmospheric for the rotational speeds of interest. The pressure and $\mathrm{CO}_{2}$ controller has to compensate for small leakages of air into the closed loop of the facility, limiting the maximum $\mathrm{CO}_{2}$ concentration to $50 \mathrm{wt} \%$.

\section{Compressor stage and operating point definition}

The investigated high-speed centrifugal compressor stage is a state-of-the-art industrial design for turbocharging applications. The stage consists of an impeller with 9 main and 9 splitter blades and the downstream diffusor consists of 18 evenly spaced vanes, acting as source of forced blade vibration.

The study has been conducted for impeller main blade mode 4 for three volume flow rates and two working fluid compositions. Resonance conditions are achieved at $75 \%$ of the compressor's design rpm. Higher modes exceed the rotational speed limitations of the facility and lower modes were found to be not critical to high cycle fatigue failures due to low blade forcing that occurs at volume flow rates and pressure ratios $<50 \%$ of the design point.

The two working fluid compositions applied to the system consist of pure air and an air- $\mathrm{CO}_{2}$ mixture with $46 \mathrm{wt} \%$ of $\mathrm{CO}_{2}$. The differences in working fluid properties are shown in Table 1 . Changes in the specific gas constant, specific heat and heat capacity ratio lead to changes in the compressor performance. At constant rotational speed the circumferential Mach number at the impeller exit increases by $11 \%$, and an increase in pressure ratio is expected. This also results in an increase of the volumetric flow rate at choked conditions in the diffuser throat area, which is illustrated in Figure 4 of the performance map. Further information about this behaviour can be obtained from (Casey and Rusch, 2014), which provides a one-dimensional approach for determining the maximum flow capacity of the stage for a choked diffuser.

The differences in the performance characteristics require the selection of comparable operating points. A specific percentage of the choked flow rate was used for comparison. Near choked (NC) conditions are defined by a volumetric flow rate of $97.5 \%$ of the choked volumetric flow rate for the applied gas. Near design (ND) conditions are subsequently defined at $90 \%$ and near stall (NS) at $80 \%$ choked volumetric flow rate. The inlet pressure for the $\mathrm{CO}_{2}$ mixture was adapted to achieve same gas density as for pure air at the compressor inlet. This

Table 1. Ratio of gas properties for constant compressor inlet density.

\begin{tabular}{|l|l|}
\hline $\mathrm{Mu}_{\text {Mix }} / \mathrm{Mu}_{\text {Air }}$ & 1.11 \\
\hline$\gamma_{\text {Mix }} / \gamma_{\text {Air }}$ & 0.96 \\
\hline $\mathrm{P} E_{\text {Mix }} / P E_{\text {Air }}$ & 0.84 \\
\hline
\end{tabular}


decision was made to make optimal use of the available power of the facility and to maximize the vibrational response amplitudes.

\section{Blade vibration measurements}

Each of the 9 impeller main blades has been instrumented with three strain gauges at locations with maximum surface strain for mode 1, 3 and 4, using the procedure presented by (Szwedowicz et al., 2002). Three splitter blades have been instrumented with a strain gauge as well, leading to a total number of 30 sensors. All strain gauges are recorded simultaneously by the in-house data acquisition system, which is located inside a spinner, mounted on the impeller hub. The impeller with the acquisition system is shown in Figure 2. It consists of 8 circular acquisition cards, stacked on top of each other and powered by a battery pack. The system consists of no moving parts leading to a maintenance free operation and no struts are distorting the upstream flow. Similar acquisition cards have been introduced by (Rebholtz et al., 2015) to measure unsteady blade surface pressure on a rotating axial turbine disk.

The measurement chain of each SG has been calibrated to elude deviations in the various current sources and signal amplifiers. This has been achieved by placing well-known resistors in parallel to the strain gauges. The resulting drop in equivalent resistance has been linearly correlated to the change in the ADC converter's digital level recording.

Measurement uncertainty estimation with (GUM, 1995) has been conducted for each strain gauge individually. The measurement uncertainty is calculated to be $6.5 \%$ of the measured response amplitude. Noise to signal ratio during calibration is $1.42 \mathrm{e}-2$ and is expected to drop further during operation since the closed loop would shield the acquisition chain from ambient electromagnetic noise. Fourier transformation of resonance crossing measurements show that the average off-resonance noise carpet is 2 orders of magnitude lower than the resonance amplitudes and is therefore neglected. Sensitivity of the boards varies between 1e2 and 1e3 digital levels per megapascal surface strain. The sampling frequency is set to resolve the resonance of interest with 14 samples per period.

A transient measurement approach has been used to avoid exposing the compressor blades to continuous resonance conditions. A constant sweep rate of $1 \mathrm{rps} / \mathrm{s}$ has been chosen to achieve quasi-steady state blade response as suggested in (Ewins, 2000).

\section{Unsteady CFD simulations}

The numerical domain consists of a $40^{\circ}$ segment modelling one main and one splitter blade in the impeller, as well as 2 diffuser passages. The computational domain includes the blade filets and the tip gap height is set to

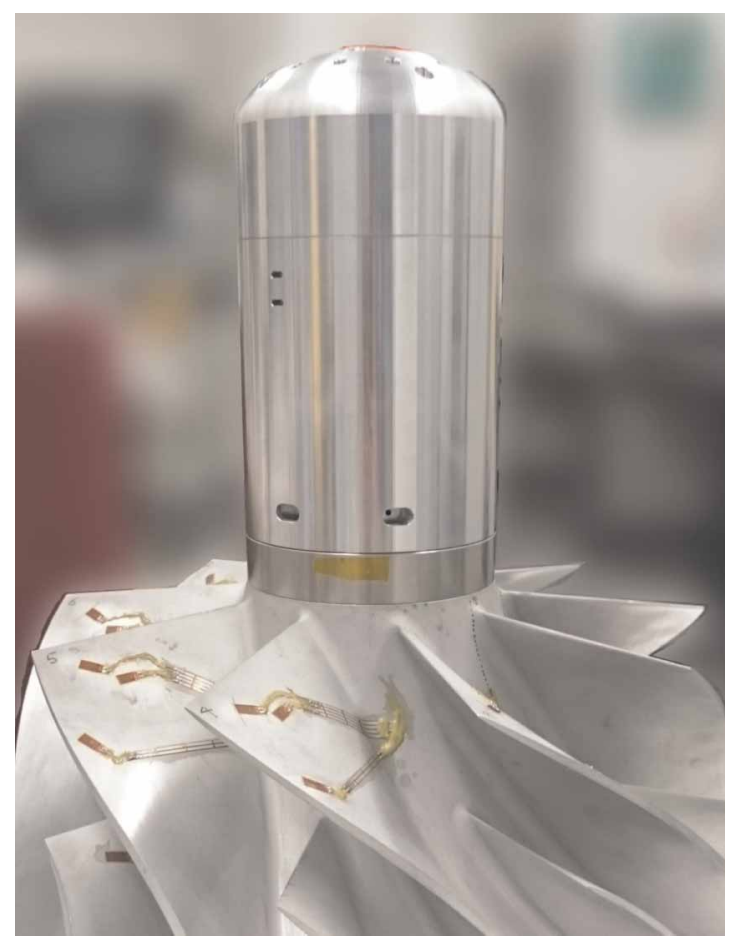

Figure 2. Instrumented impeller with strain gauges and measurement system. 
values measured in the test rig. The numerical inlet is located 4.5 impeller blade heights upstream the impeller and the domain follows the geometry of the acquisition system as well as a contraction of the facility's inlet pipe. To reduce the influence of the numerical outlet, the position of the boundary has been extended by 4 diffuser vane heights downstream of the diffuser exit and has been pinched to avoid numerical back flow. The segment consists of a structured mesh with 7 million elements where 2 million elements are used to model the diffuser area and 5 million elements for the impeller and its upstream geometry.

Temporal resolution is $0.5^{\circ}$ rotation per time step, which leads to 40 time steps per vane passing to resolve the unsteady impeller diffuser interaction. The commercial solver ANSYS CFX 18.2 has been used to conduct the numerical flow field investigation. High resolution schemes were used and for turbulence the k-omega SST model (Menter, 1992) was applied.

Rotational speed is set to the resonant condition of the experiment. The inlet boundary conditions are defined as the total pressure and total temperature measured in the facility. The operating points are defined relative to the choked mass flow rate, similar to the experimental approach. The outlet boundary condition is static pressure that was tuned to match the target flow rate.

As convergence criteria for the unsteady CFD simulation serves the generalized force $F_{G}$ acting on the main blade as defined in Equation 1. $F_{G}$ is calculated for each full numerical revolution of the impeller and compared to previous revolutions. Convergence is defined when the time dependent variation of the force is within a $10 \%$ confidence interval around its cumulative average. The generalized force $F_{G}$ and the visualization of the integrant of Equation 1 will be used to determine the surface regions responsible for the changes in vibratory response amplitudes.

\section{Harmonic FSI simulations}

The impeller is represented as a $40^{\circ}$ segment including one main and one splitter blade modelled by quadratic tetrahedrons. The mesh consists of 120,000 elements and has found to be sufficient after a refined mesh with 500,000 elements resulted in a $1 \%$ change in response amplitude.

The simulations are set up with Abaqus 6.14 and are based on the modal superposition approach. Cyclic symmetry is applied to the rotational periodic boundaries. Centrifugal load and static surface pressures are applied to the model to achieve a pre-stressed state. In a following step the eigenmodes of interest are extracted, and in a final step the resonance response is predicted. Excitation is achieved by applying the frequency content of the blade surface pressure at the diffuser vane passing frequency to the blades obtained by the unsteady CFD simulations. The applied modal damping has been determined by fitting the SDOF model to the strain gauge measurement results. The normalized mode shape magnitude of mode 4 is presented in Figure 3. It is a view of the main blade pressure side with the leading edge pointing to the left. The maximum deformation is highlighted in red colours and is located at the tip region of the impeller leading edge. Two further local maxima are located mid span at the leading edge and mid stream at the tip.

\section{Generalized force}

In the scope of this work, the generalize force $F_{G}$ is used as convergence criteria for the unsteady CFD simulations and in the subsequent analysis to explain the cause for the observed resonance response amplitudes. $F_{G}$ is defined as:

$$
F_{G}=-\oiint \phi \cdot\left(P_{u} n\right) d A
$$

where $\phi$ is the normalized shape of main blade mode 4 in complex notation, obtained from the harmonic FSI setup. $P_{u}$ represents the complex surface pressure at the vane passing frequency and $\boldsymbol{n}$ is the surface normal. The

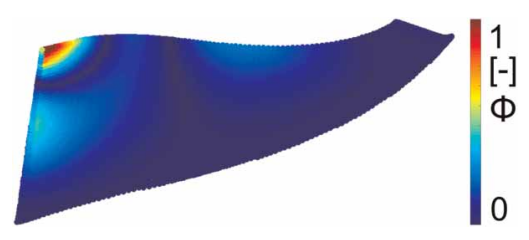

Figure 3. Normalized mode shape magnitude of impeller main blade mode 4. View at pressure side. 
generalized force is obtained by integration over the blade surface area $A$. To assess the excitation, the amplitude of $F_{G}$ as well as the phase information are of relevance. Therefore it is recommended to consider the real and imaginary components of $F_{G}$ separately. Assuming resonance and low system damping, the system response $\phi$ and the exciting force $F_{G}$ are $90^{\circ}$ phase shifted. $\phi$ can be expressed in a state of minimum deflection, where it becomes purely imaginary. Subsequently $P_{u}$ can be rotated to maximize the real component of $F_{G}$. This way the real components of $F_{G}$ and the integrand of Equation 1 represent the maximum possible excitation and the imaginary components, which are out of phase, are not able to excite the resonance.

\section{Results and discussion}

This section evaluates the differences in compressor performance between the investigated operating points and the two working fluids. The strain gauge measurements of the mode 4 resonance crossings allow estimating the influence of damping and forcing to the resonant response amplitudes. The highest difference in resonance amplitude between air and $\mathrm{CO}_{2}$ mixture appears at near choked conditions. These two points are investigated in detail by determining the generalized force distribution on the blade to highlight the cause for the change in resonant amplitude. These effects are linked to wave propagation effects in the flow field.

\section{Compressor operating points}

In turbocharging applications the compressor operates in a wide range of rotational speeds, which are defined by the demand of the combustion engine. The main blade mode 4 resonance crossing for this compressor configuration appears at a representative operating condition of $75 \%$ design speed $n_{\text {ref }}$. Figure 4 shows the performance map for the two investigated working fluids at different flow settings, as defined in the methodology section. The plot visualizes the total pressure ratio $\pi_{0}$ over the temperature corrected volumetric flow rate $\dot{V}_{298}$ at the compressor inlet at the rotational speed for the mode 4 resonance. The corrected volume flow rate at compressor inlet is defined as

$$
\dot{V}_{298}=\dot{\boldsymbol{V}} \sqrt{\frac{298 \boldsymbol{K}}{\boldsymbol{T}_{0}}}
$$

Comparing the two gas compositions with each other shows that the $\mathrm{CO}_{2}$ mixture results in an increase in pressure ratio by $22 \%$.

Below design speed the compressor usually chokes in the diffuser throat area, and the $\mathrm{CO}_{2}$ mixture results in a $9 \%$ greater choked flow rate as compared to air. The increased pressure ratios and flow rates for the mixture are

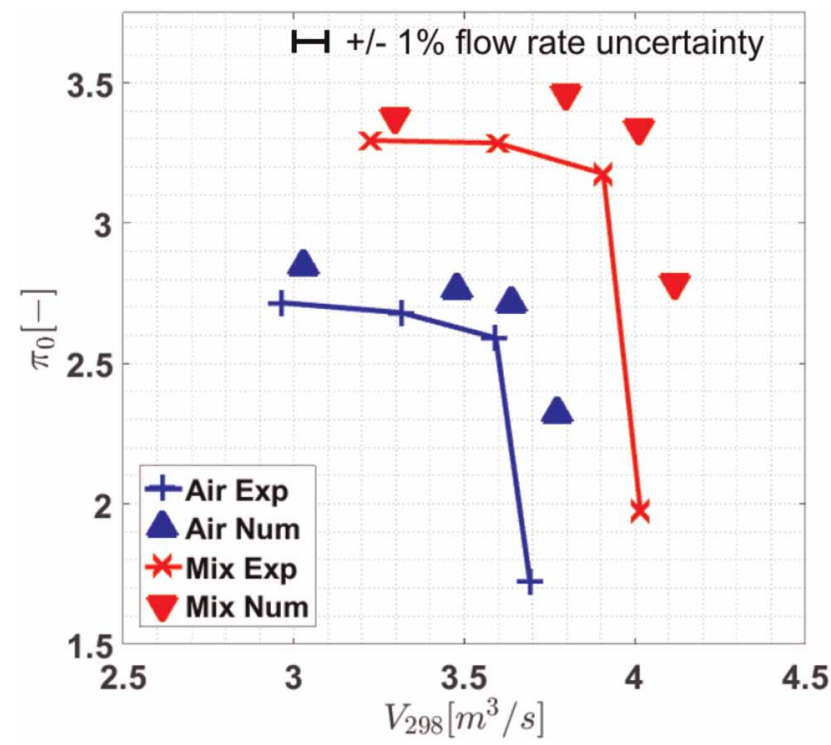

Figure 4. Normalized compressor performance for air and air $-\mathrm{CO}_{2}$ mixture. CFD results are represented as single points. 
correlating with the changes of circumferential Mach number and working fluid properties (Casey and Rusch, 2014).

The choked measurement points were measured with the throttle fully opened. The low pressure ratios at this points are representing the resistance of the closed loop system. The investigated operating points are defined relative to the choked volume flow as described in the methodology section.

The time averaged CFD results are shown as single points in Figure 4. The CFD over predicts the choked flow rate for both gases by $2 \%$ while the measurement uncertainty of the facility is $\pm 1 \%$. The total pressure ratios of the CFD results are around 5\% above the measured values since the CFD model does not include the collector geometry of the facility. The total pressure losses generated between the diffuser exit and the measurement plane ("Stage Outlet O") in Figure 1 are not captured by the numerical model, but the differences are in agreement with literature data on losses generated by compressor volutes (Cumpsty, 1989). The time resolved volumetric flow of the unsteady flow simulations oscillate around an average value, reaching from $0.5 \%$ at the near choke operating conditions and reach up to $4 \%$, close to the near stall operating conditions. This behaviour is caused by mismatch in flow incidence at part load conditions.

\section{Contribution of blade damping and forcing to resonance response}

The resonance crossing of mode 4 excited by the vaned diffuser is shown in Figure 5 where the amplitude spectrum of an on-blade strain gauge is plotted over a variation in rotational speed. The information obtained from the strain gauges is used to determine the maximum resonant response amplitudes $\sigma$ for all blades at constant inlet density. The response amplitudes averaged over the impeller blades is shown in Figure 6. Air at the near choked operating condition is used as reference value $\sigma_{\text {ref }}$ to normalize the results. The $x$-axis shows the volume flow rate relative to choked conditions. The bars indicate the standard deviation in response amplitude between the impeller blades and reach from $14 \%$ to $31 \%$ of the measured response amplitude. Mistuning effects and tolerances in SG instrumentation are a systematic contributor to the deviation and are superimposed by stochastic variations in the resonance response and by measurement uncertainties. Repeatability tests on an operating point with a different inlet pressure showed equal contribution of systematic and stochastic variations. The single points shown in the plot are obtained by FSI simulations and are discussed in subsequent sections.

The measurement results for air show a continuous decrease in amplitude from $100 \%$ at near choke to $49 \%$ at near stall conditions. The response amplitudes for the $\mathrm{CO}_{2}$ mixture are lower and are showing a different trend with flow rate. Maximum response amplitude is at $49 \%$ of the reference value and reduces down to $29 \%$ at near stall and $25 \%$ at near choke conditions. The generally lower resonance response amplitudes for the mixture can reduce the risk of HCF failures for this mode. The variations in resonance amplitudes depend on the exciting force and the damping on the blades. The contribution of these two variables is determined by assuming the resonance crossings follow the response of a SDOF system.

The critical damping ratios $\zeta$ of the resonance crossings have been estimated by fitting a SDOF response to the signal shape of the resonance crossing. Details of this procedure can be found in (Craig and Kurdila, 2006) and have been applied to a radial compressor by (Kammerer and Abhari, 2008). The results presented in Figure 7 have been normalized with the damping, obtained at near choke conditions and air. The error bars

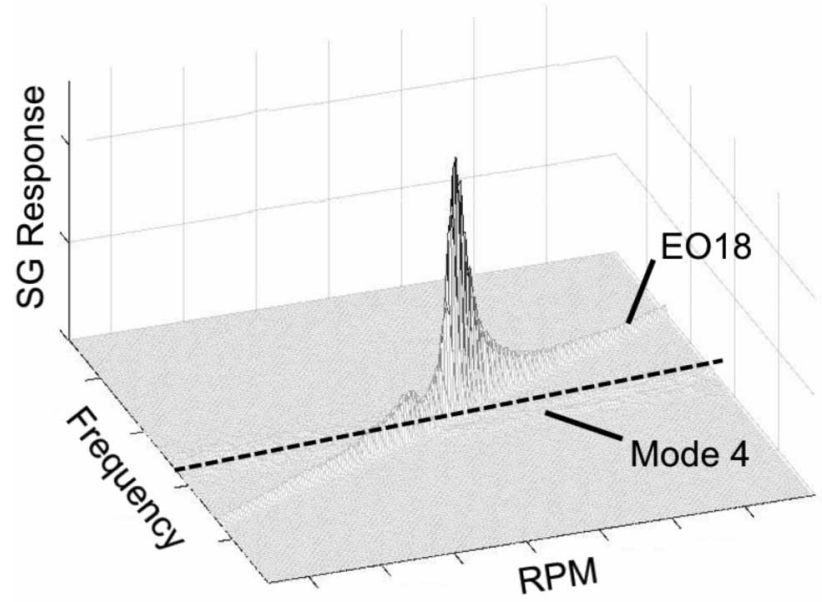

Figure 5. Campbell plot of measured mode 4 resonance crossing. 


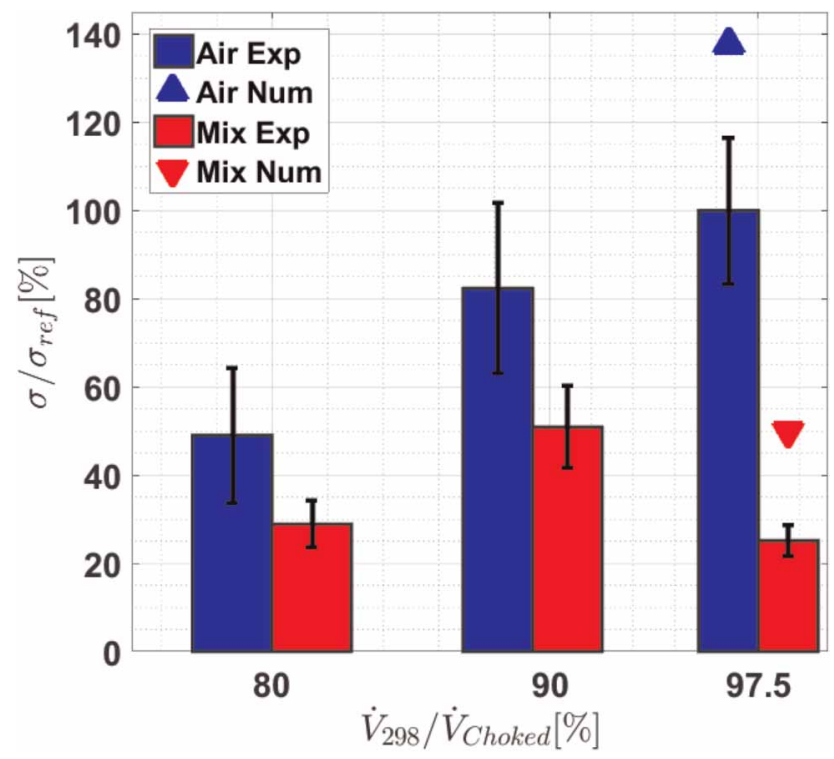

Figure 6. Measured and simulated blade vibratory response amplitudes of mode 4.

indicate the repeatability of $\pm 15 \%$ for the damping estimation based on multiple measured resonance crossings at an operating point with a different inlet pressure.

Both gases possess the highest damping values at near choked operating conditions and are not distinguishable from each other. Both show a decrease in damping ratio with a decrease in flow rate. At near stall conditions air is at $67 \%$ and mixture at $81 \%$ of the reference damping. The mixture shows a tendency of slightly higher damping values than air but still within the uncertainty range. It indicates that flow rate has a higher impact on the damping properties than the gas properties.

The knowledge of resonance response amplitudes from Figure 6 and the critical damping ratios in Figure 7 allow estimating the influence of the exciting force $F$. The linear correlation given in Equation 3 is based on a SDOF model at resonance.

$$
F \sim \sigma \cdot \zeta
$$

The results of evaluating Equation 3 for the different test cases are presented in Figure 8 with the force for air at near choke as reference. The uncertainty is estimated to be $\pm 21 \%$ and is obtained by combining the repeatability

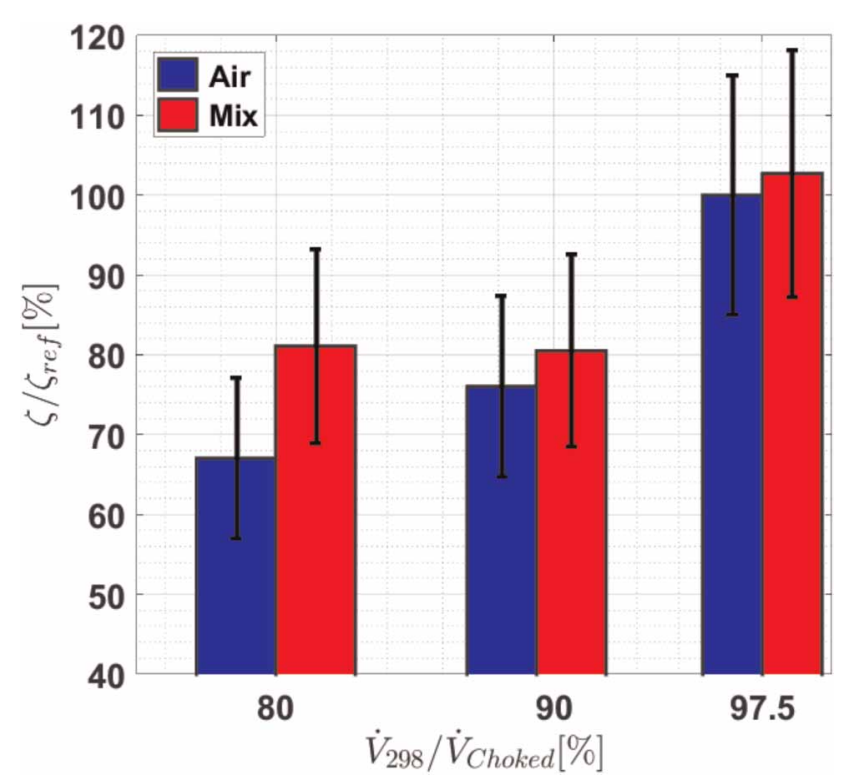

Figure 7. Critical damping ratios of mode 4. 


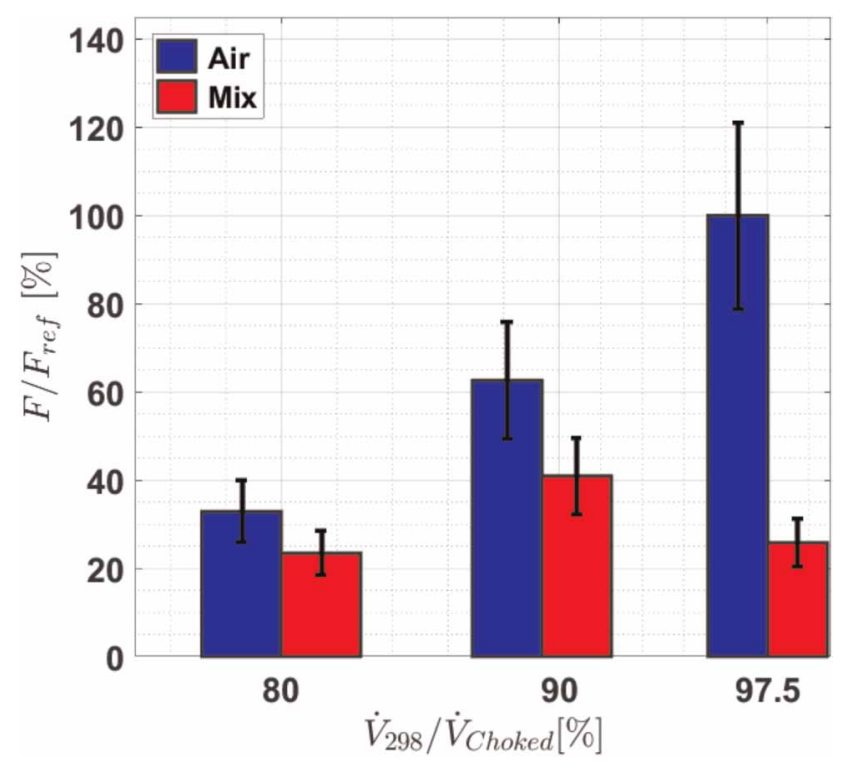

Figure 8. Forcing acting on main blade mode 4.

effects of the response amplitude and the damping estimation. The forcing appears to be the main contributor to the observed changes in response amplitudes since the plot reassembles the resonance response pattern. At near choked conditions both gases possess similar damping and the observed factor of 4 difference in response amplitude is caused by the exciting force. At the near stall conditions the differences in response amplitude between the gases are caused equally by changes in damping and forcing. Forcing for air decreased continuously with flow rate to $33 \%$ to compensate for reduced damping values, while the trend for the $\mathrm{CO}_{2}$ mixture is unsteady.

In the subsequent numerical investigation the change in forcing will be discussed between air and the $\mathrm{CO}_{2}$ mixture at near choked operating conditions. The strain gauge measurements are able to determine the change in forcing but are not able to determine blade areas responsible for the changes.

\section{Harmonic FSI response analysis}

Before interpreting the CFD blade pressure distribution for the generalized force and its impact on excitation it is recommended to estimate the accuracy of the CFD simulations by comparing the vibratory response measurements with results of a harmonic response analyses.

Figure 6 shows the measured response amplitudes together with the outcome of the FSI simulation for NC conditions.

The simulation results are over predicting the response amplitudes for both operating conditions. The simulation results for the mixture are over predicted by $100 \%$ and for air the FSI approach is over predicting the response amplitude by 38\%. It cannot be determined clearly if the differences are dominated by offset or scaling effects. Even though the absolute amplitudes are over predicted the amplitude ratio between both cases is 2.8 . This trend is consistent with the ratio of 4 observed in the SG measurements and therefore it is possible to explain the differences in excitation based on the CFD results.

\section{Unsteady CFD and generalized force distribution}

The aim of the flow simulations is to obtain information about the spatially and time resolved blade pressure to investigate the forcing. The frequency components responsible for exciting the mode 4 are equal to the diffuser vane passing frequency and are extracted by Fourier transformation. Authors in the past have described the unsteady forcing on the compressor blades caused by the downstream diffuser vanes as upstream traveling waves. Maximum amplitude was found to be at the trailing edge area of the compressor and dropping in upstream direction (Gould et al., 2007; Walton and Tan, 2016). This will lead to a time dependent interaction between blade deflection and the local blade surface pressures. It was also found to be sensitive to the involved mode, geometries and operating point and thus difficult to predict.

The generalized pressure distribution, defined as the integrand of Equation 1, is presented in Figures 9 and 10 for air and the $\mathrm{CO}_{2}$ mixture at near choked conditions. The images show the magnitude of the complex 

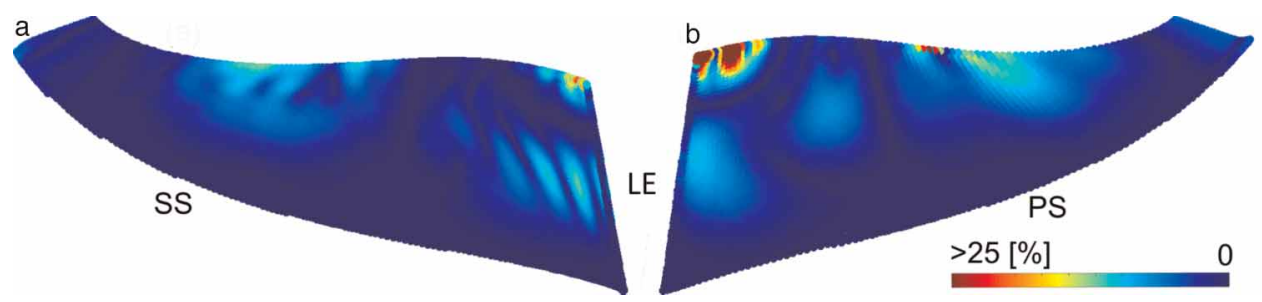

Figure 9. Generalized pressure amplitude distribution for mode 4 and air. Impeller main blade suction side (a) and pressure side (b).

distribution, representing the excitability of the blade. The mode shape can be interpreted as the sensitivity of the blade to interact with the local force caused by the pressure unsteadiness. The colour range is consistent for all the plots to allow a qualitative comparison. Zero amplitude is visualized in blue and maximum in red colour. In all of the presented figures the pattern reassembles the mode shape. The sensitivity is dominating over the pressure unsteadiness, which is highest at the trailing edge area, in proximity to the diffuser. Comparison of both gases with each other reveals increased pressure unsteadiness for the $\mathrm{CO}_{2}$ mixture at the trailing edge region (Figure 10b). Figure 9b shows the pressure side of the main blade for air and reveals higher generalized pressure at the impeller leading edge region and mid stream at the tip region where the mode shape possesses deflection. Both, the amplitude and the relevant area are increased, compared to the mixture (Figure 10b). A similar trend is visible at the main blade suction side in Figures $9 \mathrm{a}$ and 10a. The relatively rigid trailing edge area is contributing to the generalized pressure since it possesses the highest unsteady blade pressure amplitudes (Zemp and Abhari, 2012).

Local excitation on the blade is a combination of force on pressure and suction sides $\left(F_{G P S}+F_{G S S}\right)$. In addition to the amplitude, the phase of the local force is also of relevance for the excitation. With reference to the methodology section, at resonance the blade response $\phi$ and the global excitation force $F_{G}$ are $90^{\circ}$ phase shifted. The generalized force can be expressed under the condition of maximum excitation. The resulting generalized force can be decomposed into its real and imaginary components. Within this work the real components indicate the maximum possible excitation and the imaginary components are out of phase and unable to interact with the resonance. The results of this approach are presented in Figure 11. Equation 1 has been integrated over the blade height in 5\% streamwise increments, starting from $0 \%$ at the impeller leading edge. The generalized force for air at near choked conditions $F_{G \text { ref }}$ has been used for normalization in both cases. Therefore the sum of the real components along the streamwise direction for air will be $100 \%$ and for the mixture it was found to be $34 \%$. These numbers are in agreement with the FSI simulation results presented in the previous section.

The generalized force distribution for air is presented in the Figure 11a. The relatively rigid trailing edge area between 90 and 100\% streamwise location is contributing around $10 \%$ to the generalized force and is dominated by the local high unsteady pressure amplitudes. The pressure amplitudes decrease in the upstream direction, and together with the local phase distribution, it results in low and partially negative generalized force components between 60 and 90\% streamwise. For air the midstream area (25-60\% streamwise), where the mode possesses deflection, is the main contributor to the generalized force with approximately $65 \%$ of the overall amplitude. The leading edge area ( $<25 \%$ streamwise) possesses low unsteady pressure amplitudes but contributes $25 \%$ to the generalized force due to high mode deflections.

The $\mathrm{CO}_{2}$ mixture, Figure $11 \mathrm{~b}$, shows for the trailing edge area an increase in generalized force to $14 \%$ of the reference value. This is caused by higher unsteady pressure amplitudes and shifts the first negative real
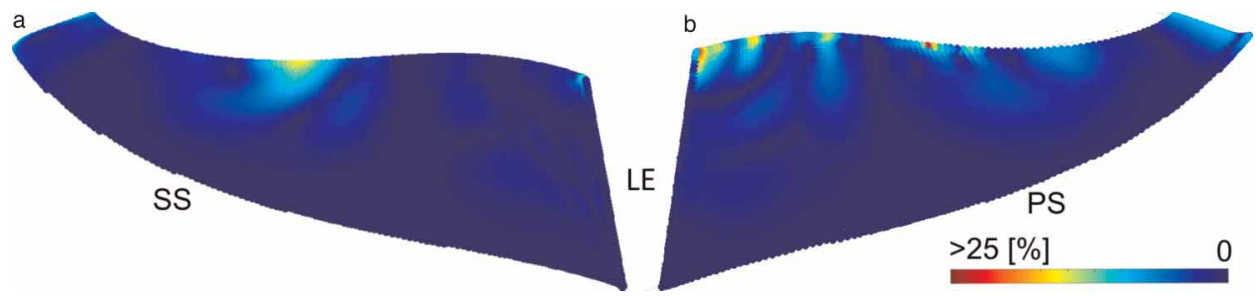

Figure 10. Generalized pressure amplitude distribution for mode 4 and air- $\mathrm{CO}_{2}$ mixture. Impeller main blade suction side (a) and pressure side (b). 

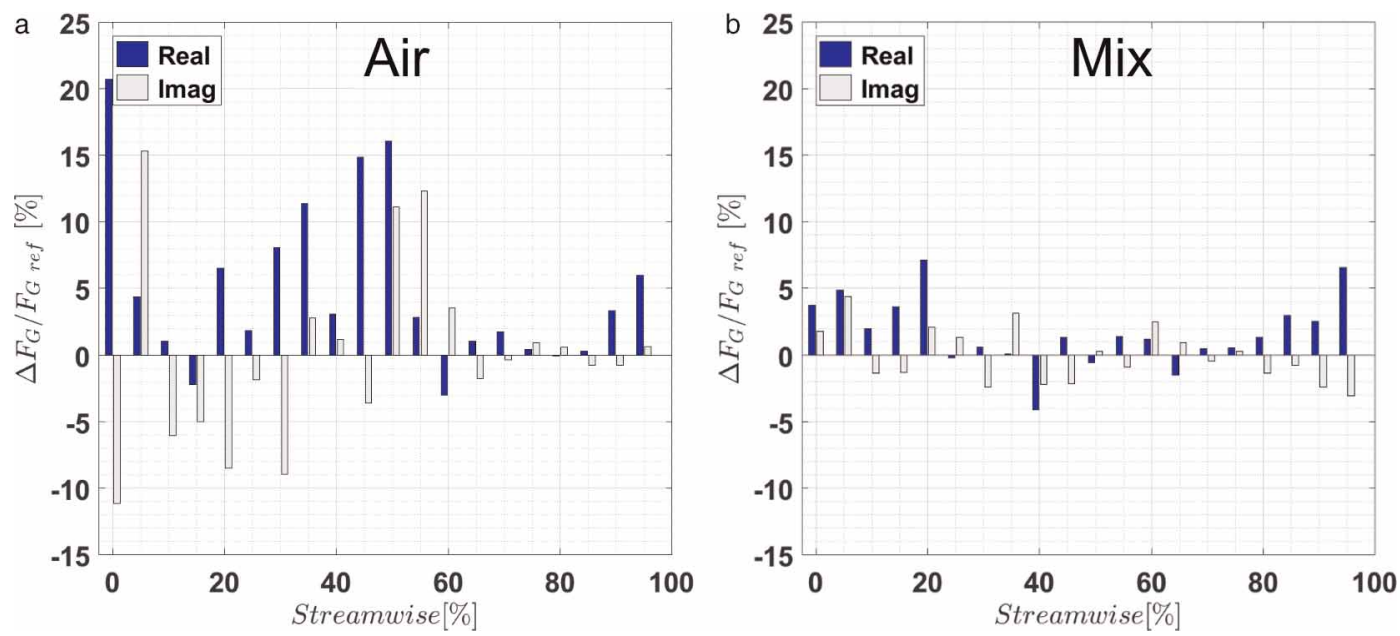

Figure 11. Real and imaginary components of the generalized force along streamwise direction. For air (a) and air- $\mathrm{CO}_{2}$ mixture (b).

component to $70-85 \%$ streamwise location. The $30-80 \%$ streamwise area was found to be contributing to the reduced forcing with the $\mathrm{CO}_{2}$ mixture. The generalized force amplitudes are lower in amplitude and are dominated by negative real components, counteracting the excitation in this area by $-7.4 \%$. In this case the leading edge area $(<25 \%$ streamwise $)$ contributes the most to the forcing with around $25 \%$ of the reference value. The overall excitation in the $<25 \%$ streamwise area comparable to air. In the case of the mixture the amplitudes are lower but the phase distribution is more beneficial to the excitation.

To explain the cause of these differences the modal content of the static pressure in the flow is visualized in Figure 12 at a 95\% spanwise location. The real components of the static pressure are calculated under the condition of maximum excitation and shown in a logarithmic scale for improved visualization. Negative values are visualized in blue and positive values in red.

The highlighted area indicates the midstream region where the mode possesses deflection. It can be seen that for air (Figure 12a) the wave pattern is leading to a high blade load. The $\mathrm{CO}_{2}$ mixture in Figure 12b possesses a different wave pattern with local elimination of the load with respect to the mode shape. The load depends on wave propagation effects in upstream direction. The potential field from the diffuser can be seen in the upper part of the figures and is alternating around the blade trailing edge area. This leads to pressure waves travelling in upstream direction. The speed of propagation and therefore the spatial wave length depends on the local speed of sound and the opposing flow Mach number. Further influence is caused by the splitter blade leading edge area where the flow channels merge and also by the main blade leading edge. The pattern is highly case dependent and a small change in the working fluid and operating point can change the pressure field with respect to the mode shape. In the case of the $\mathrm{CO}_{2}$ mixture, the pressure field is not well aligned to the mode shape, leading to a reduced forcing and low resonance amplitudes. These observations suggest co-optimizing the compressor design with respect to minimizing the generalized force for critical blade modes. This will be relevant for turbo charging applications where due to the big range of rotational speeds a resonance free operation cannot be ensured.

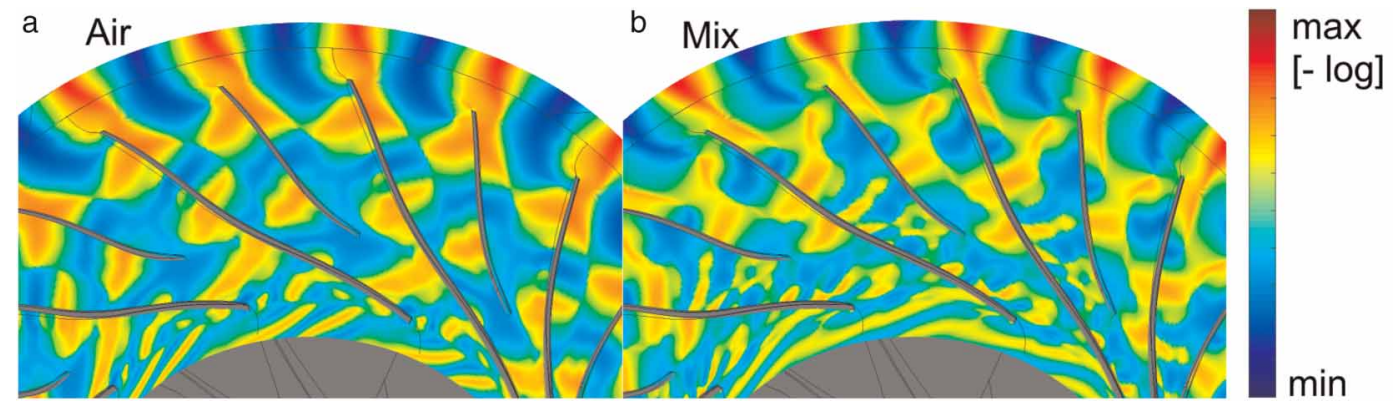

Figure 12. Real components of the static pressure field at $95 \%$ spanwise location in logarithmic scale. For air (a) and air- $\mathrm{CO}_{2}$ mixture (b). 


\section{Conclusions}

The resonance response behaviour of an industrial design centrifugal compressor stage has been investigated for two different working fluid compositions. This allows changing the system's damping and forcing without influencing the modal response. On-blade strain measurements were conducted to capture the resonance response amplitude of impeller main blade mode 4 excited by the vaned diffuser. Measured resonant response amplitudes for air are up to a factor of 4 higher than for the $\mathrm{CO}_{2}$ mixture at near choke conditions. This can pose a risk of high cycle fatigue failures and has to be considered in the design process of the compressor.

Fitting a SDOF oscillator to the measured resonance crossings allowed determining the contribution of forcing and damping to the measured response amplitudes. At the near choked operating points similar damping values for air and the air- $\mathrm{CO}_{2}$ mixture are observed. In this case the difference in resonance amplitude is dominated by changes in the unsteady blade forcing. For lower flow rates the differences in the damping increases but the forcing remains the main contributor to the changes in amplitude.

Unsteady CFD simulations of the compressor stage were used to obtain time-resolved blade pressure information, serving as input boundary conditions for harmonic FSI simulations. The FSI setups over-predict the resonance amplitudes at the near choked operating conditions, but are able to capture the trend in response amplitudes and thus the CFD model captures the responsible flow effects. This justifies the usage of the generalized force distribution to identify the blade areas responsible for the changes in the forcing.

It was found that, even though the impeller diffuser interaction causes maximum unsteady blade pressure at the impeller trailing edge area, it contributes less than $15 \%$ to the generalized force for both working fluids. The excitation is dominated by the areas where the mode possesses deflection and thus by the sensitivity of the blade.

Two effects were found to dominate the changes in resonance response between air and the $\mathrm{CO}_{2}$ mixture at near choked conditions. First, reduced amplitudes of unsteady blade pressure in the sensitive areas and second, negative forcing components which are locally counteracting the excitation. This depends on the local blade pressure and phase on both sides of the blade combined with the vibration direction if the blade.

The origins of these differences were found in wave propagation effects originating from the impeller trailing edge area, propagating in upstream direction and their interactions in the blade leading edge areas of splitter blade and main blade. The resulting generalized force is sensitive to the compressor operating conditions and depends on how well the excitation pattern matches with the mode shape. Based on these observations a co-optimization of flow dynamics and structural dynamics to minimize excitation of critical resonances is suggested. Especially in turbocharging applications where resonance free operation cannot be ensured, the resonance crossings can be optimized to appear at operating conditions where the forcing pattern matches the mode shape less.

\section{Nomenclature}

A Blade surface area

ADC Analog-to-digital converter

CFD Computational fluid dynamics

$\mathrm{CO}_{2}$ Carbon Dioxide

CCS Carbon capture and storage

FSI Fluid-structure interaction

$F \quad$ Exciting force of a SDOF system

$F_{G} \quad$ Generalized force

ETH Swiss Federal Institute of Technology

$f \quad$ Frequency

IDI Impeller-diffuser interaction

Imag Complex imaginary component

LE Blade Leading Edge

$\mathrm{Mu}$ Circumferential Mach number

$n \quad$ Blade surface normal vector

$n \quad$ Rotational speed

NC Near choked flow settings

ND Near design flow settings

NS Near stall flow settings

PE Static pressure at compressor inlet 
$P_{u} \quad$ Unsteady blade surface pressure

PS Main blade pressure side

Real Complex real component

RIGI Centrifugal compressor test facility

SDOF Single degree of freedom model

SG Strain gauge

SS Main blade suction side

$T \quad$ Temperature

TE Blade Trailing Edge

$\dot{V} \quad$ Volume flow rate

$\gamma \quad$ Heat capacity ratio

$\Delta \quad$ Difference; blade PS-SS

$\sigma \quad$ Blade surface strain

$\zeta \quad$ Critical damping ratio

$\pi \quad$ Pressure ratio

$\phi \quad$ Normalized mode shape vector

\section{Additional subscripts}

0 Total quantity

Air Air, ideal gas

Mix Air and 46wt $\% \mathrm{CO}_{2}$, ideal gas

ref Reference values

\section{Acknowledgements}

Thanks go to the substantial contributions of Dr. Magnus Fischer, Dr. Marius Banica, Mr. Gerd Mundinger and Mr. Arian Röber from ABB Turbo Systems Ltd. in numerical simulations. For the intellectual support on the experimental side Dr. Peter Hoenisch from ABB Turbo Systems Ltd. is acknowledged. The Authors would like to thank ABB Turbo Systems Ltd. for the permission to publish this work.

\section{Funding sources}

The authors gratefully acknowledge the intellectual and financial support of their industry partner ABB Turbo Systems Ltd. The authors gratefully acknowledge the financial support of the Commission for Technology and Innovation (CTI).

\section{Competing interests}

Carsten Degendorfer, Reza Abhari, Klemens Vogel and René Hunziker declare that they have no conflict of interest.

\section{References}

Al-Abdulkarem A., Hwang Y., and Radermacher R. (2012). Efficiency improvement of natural gas combined cycle power plant with $\mathrm{CO}_{2}$ capturing and sequestration. ASME International Mechanical Engineering Congress and Exposition, Volume 6: Energy, Parts A and B. ASME. 21-26. https://doi.org/10.1115/IMECE2012-87436

Boncinelli P., Emini E., Bartolacci S., and Arnone A. (2007). Impeller-diffuser interaction in centrifugal compres- sors: numerical analysis of radiver test case. Journal of Propulsion and Power. 23: 1304-1312. https://doi.org/10.2514/1.27028

Botero C., Finkenrath M., Belloni C., et al. (2009). Thermoeconomic evaluation of $\mathrm{CO}_{2}$ compression strategies for post-combustion $\mathrm{CO}_{2}$ capture applications. In: ASME Turbo Expo: Power for Land, Sea, and Air, Volume 4: Cycle Innovations; Industrial and Cogeneration; Manufacturing Materials and Metallurgy; Marine. 517-526. https://doi.org/10.1115/GT2009-60217

Casey M. and Rusch D. (2014). The matching of a vaned diffuser with a radial compressor impeller and its effect on the stage performance. Journal of Turbomachinery. 136 (12): 121004. https://doi.org/10.1115/1.4028218

Craig R. R. and Kurdila A. J. (2006). Fundamentals of Structural Dynamics. John Wiley \& Sons, Hoboken, NJ.

Cumpsty, N. A. (1989). Compressor Aerodynamics. Longman Scientific \& Technical, Harlow, Essex.

El-Aini Y., deLaneuville R., Stoner A., and Capece V. (1997). High cycle fatigue of turbomachinery components - industry perspective. In: 33rd AIAA/ASME/SAE/ASEE Joint Propulsion Conference, Vol. AIAA 97-3365

Ewins D. J. (2000). Modal Testing Theory, Practice and Application, 2nd ed. Baldock, Hertfordshire, England: Research Studies Press.

Gallier K., Lawless P., and Fleeter S. (2010). Particle image velocimetry characterization of high-speed centrifugal compressor impellerdiffuser interaction. Journal of Propulsion and Power. 26 (4): 784-789. https://doi.org/10.2514/1.38663 
Gould K. A., Tan C. S., and Macrorie M. (2007, January). Characterization of unsteady impeller-blade loading in a centrifugal compressor with a discrete-passage diffuser. In: ASME Turbo Expo 2007: Power for Land, Sea, and Air. 1781-1790. American Society of Mechanical Engineers.

GUM. Guide to the expression of uncertainty in measurementa, first edition, 1993, corrected and reprinted 1995, International Organization for Standardization (Geneva, Switzerland), dtsch: Leitfaden zur Angabe der Unsicherheit beim Messen, 1. Auflage 1995, DIN V ENV 13005, 1999/06, Deutsches Institut für Normung - Beuth-Verlag

Haupt U. and Rautenberg M. (1984). Blade vibration measurements on centrifugal compressors by means of telemetry and holographic-interferometry. Journal of Engineering for Gas Turbines and Power-Transactions of the ASME. 106 (1): 70-78. https://doi.org/10.1115/1.3239553

Heo J., Kwon J., and Lee J. (2017). A study of S-CO $\mathrm{CO}_{2}$ power cycle for CSP applications using an isothermal compressor. Journal of Engineering for Gas Turbines and Power. 140: 071702. https://doi.org/10.1115/1.4038476

Kammerer A. and Abhari R. S. (2008). Experimental study on impeller blade vibration during resonance—Part II: blade damping. Journal of Engineering for Gas Turbines Power. 131 (2): 022509. https://doi.org/10.1115/1.2968870

Kielb R. E. (1998). Unsteady flows: an aeroelastic blade design perspective. In: ERCOFTAC Turbomachinery Seminar and Workshop.

König S., Petry N., and Wagner N. (2009). Aeroacoustic Phenomena in High Pressure Centrifugal Compressors - A Possible Root Cause for Impeller Failures. Texas A\&M University. Turbomachinery Laboratories. http://hdl.handle.net/1969.1/163083.

Li H., Zhang X., Liu Y., Xu F., and Zhou S. (2015). Investigation on acoustic resonance for different diffuser and effect on centrifugal compressor pipeline failure. In: ASME Turbo Expo: Power for Land, Sea, and Air, Volume 2C: Turbomachinery, V02CT44A030. https://doi.org/10.1115/GT2015-43958

Menter F. R. (1992). Improved two-equation k-omega turbulence models for aerodynamic flows. NASA TM 103975.

Metz B., Davidson O., De Coninck H., Loos M., and Meyer L. (2005). IPCC special report on carbon dioxide capture and storage. Geneva, Switzerland: Intergovernmental Panel on Climate Change. Working Group III.

Pereboom H. P., van Beek P. G., and Smeulers J. M. (2016). Experimental investigation of fluid structure interaction of impeller like disks in super critical carbon dioxide. In: ASME Turbo Expo: Power for Land, Sea, and Air, Volume 7A: Structures and Dynamics. V07AT27A009. https://doi.org/10.1115/GT2016-58032.

Rebholtz P., Mansour M., Kalfas A., and Abhari R. S. (2015). An on-board wireless multi-sensor measurement system for rotating turbomachinery Application. International Gas Turbine Congress (IGTC) 2015.

Srinivasan A. V. (1997). Flutter and resonant vibration characteristics of engine blades. ASME Journal of Engineering for Gas Turbines and Power. 119 (4): 741-775. https://doi.org/10.1115/1.2817053

Szwedowicz J., Senn S. M., and Abhari R. S. (2002). Optimum strain gage application to bladed assemblies. Journal of Turbomachinery. 124 (4): 606-613. https://doi.org/10.1115/1.1506957

Walton E. J. and Tan C. S. (2016). Forced response of a centrifugal compressor stage due to the impeller-diffuser interaction. Journal of Turbomachinery. 138 (9): 091004. https://doi.org/10.1115/1.4032838

Zemp A. and Abhari R. S. (2012). Vaned diffuser induced impeller blade vibrations in a high-speed centrifugal compressor. In: ASME Turbo Expo: Power for Land, Sea, and Air, Volume 7: Structures and Dynamics, Parts A and B. 1507-1518. https://doi.org/10.1115/ GT2012-69400

Ziegler K. U., Gallus H. E., and Niehuis R. (2003a). A study on impeller-diffuser interaction-part 1: influence on the performance. Journal of Turbomachinery. 125: 173-182. https://doi.org/10.1115/1.1516814

Ziegler K. U., Gallus H. E., and Niehuis R. (2003b). A study on impeller-diffuser interaction-part 2: detailed flow analysis. Journal of Turbomachinery. 125: 183-192. https://doi.org/10.1115/1.1516815 